\title{
Identification of microRNAome in rat bladder reveals miR-1949 as a potential inducer of bladder cancer following spinal cord injury
}

\author{
TIANYI WANG ${ }^{1,2^{*}}$, YONG LIU $^{1 *}$, WENQI YUAN ${ }^{1 *}$, LIANG ZHANG $^{3}$, YANJUN ZHANG $^{1}$, \\ ZHIJIE WANG ${ }^{4}$, XIANHU ZHOU ${ }^{1}$, HENGXING ZHOU ${ }^{1}$, TIANCI CHU ${ }^{1}$, YAN HAO ${ }^{1}$,

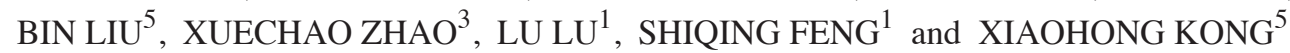

\begin{abstract}
${ }^{1}$ Department of Orthopedics, Tianjin Medical University General Hospital, Tianjin 300052; ${ }^{2}$ Department of Orthopedics, The 266th Hospital of The Chinese People's Liberation Army, Chengde, Hebei 067000; ${ }^{3}$ Department of Orthopedics, The Second Hospital of Tianjin Medical University, Tianjin 300211; ${ }^{4}$ Department of Paediatric Internal Medicine, Affiliated Hospital of Chengde Medical College, Chengde, Hebei 067000;

${ }^{5}$ School of Medicine, Nankai University, Tianjin 300071, P.R. China
\end{abstract}

Received July 7, 2014; Accepted March 20, 2015

DOI: $10.3892 / \mathrm{mmr} .2015 .3769$

\begin{abstract}
The costs of spinal cord injury and its complications are high in personal, social and financial terms. Complications include bladder cancer, for which the risk is $16-28$ times higher than that of the general population, There is currently little consensus regarding the cause of this discrepancy. As microRNAs are stable biomarkers and potential therapeutic targets of cancer, the present study aimed to explore the underlying mechanisms of this phenomenon by examining changes in the microRNAome. Rats were used to produce models of spinal cord injury. Microarrays and bioinformatics were used to investigate the cancer-associated microRNAs that are upregulated in rat bladders following spinal cord injury. In order to validate the results, quantitative reverse transcription-polymerase chain reaction (qRT-PCR), western blotting and immunohistochemistry were performed. The expression of miR-1949 was found to be deregulated and abundant in the rat bladder following spinal cord injury. Bioinformatics demonstrated that retinoblastoma 1, which is involved in tumorigenesis, is a target gene of miR-1949. qRT-PCR, western blotting and immunohistochemistry confirmed the results of the microarray analysis. In addition, it was shown that miR-1949 expression was not influenced by aging. Furthermore, the expression of miR-1949 was stable
\end{abstract}

Correspondence to: Professor Shiqing Feng, Department of Orthopedics, Tianjin Medical University General Hospital, 154 Anshan Road, Heping District, Tianjin 300052, P.R. China

E-mail: professorfengsq@163.com

Professor Xiaohong Kong, School of Medicine, Nankai University, 94 Weijin Road, Tianjin 300071, P.R. China

E-mail: professorkxh@126.com

*Contributed equally

Key words: spinal cord injury, bladder cancer, microRNA, microarray, tumorigenesis, retinoblastoma 1 until the third month following spinal cord injury, after which it significantly increased. If this increase was prolonged, the expression of retinoblastoma 1 may decline to a carcinogenic level. The present study suggests a role for miR-1949 in the translational regulation of retinoblastoma 1 and in subsequent bladder tumorigenesis following spinal cord injury.

\section{Introduction}

MicroRNAs (miRNAs) are a family of endogenous and noncoding RNAs that regulate gene expression by inhibiting mRNA translation or inducing mRNA degradation, usually by binding to the 3'-untranslated region of target mRNAs (1-3). In terms of their use as biomarkers or therapeutic targets, miRNAs are more stable than mRNAs, which are sensitive to degradation (4). The importance of miRNAs has been focused upon during the past decade, and their dysregulation has been identified as a feature of tumorigenesis.

Spinal cord injury (SCI), which commonly occurs as a result of trauma, is a significant cause of disability (5). The cost is high in personal, social and financial terms. In addition to motor and sensory dysfunction, patients with SCI are also at risk of developing multiple complications, such as bladder cancer, which may be induced by a variety of mechanisms (6-9). The incidence of bladder cancer appears to be increased following SCI; a number of retrospective studies have reported that the risk of bladder cancer risk in patients with $\mathrm{SCI}$ is $16-28$ times higher than that of the general population $(10,11)$. Numerous risk factors have been proposed to account for this increased incidence. However, there is currently little consensus regarding the risk factors for bladder cancer in patients with SCI. In a study involving 43,561 patients with SCI, it was concluded that long-term indwelling catheterization is a risk factor for bladder cancer in patients with SCI (12). Other risk factors identified for bladder cancer in patients with SCI, include urinary tract infections (UTI) and the nature of the neurogenic bladder itself $(12,13)$. However, no studies have focused on the role of miRNAs. 
After consulting a high number of studies, it was apparent that the majority of reports regarding bladder cancer following SCI are derived from epidemiological studies. To the best of our knowledge, the present study is the first to examine this phenomenon through identification of changes in the miRNA expression profile (the 'microRNAome') (14).

The Rb1 gene, located on chromosome 13q14, was the first tumor suppressor gene to be isolated (15). The Rb1 protein is a nuclear phosphoprotein, which is involved in a number of pathways of bladder cancer, either at its initiation or during its progression, including cell-cycle regulation and apoptosis $(16,17)$. Previous studies have confirmed that alteration or deletion of the Rb1 protein is observed in human bladder cancer, suggesting that the Rbl gene is critical in bladder tumorigenesis (16). In addition, tumorigenicity may be suppressed by transfecting the Rbl gene into bladder cancer cells (16). Therefore, the aims of present study were to determine the miRNA expression of rat bladder tissues following SCI, and the possible association between miR-1949, Rb1 and the development of bladder cancer.

\section{Materials and methods}

Ethics statement. Seventy-two adult female Wistar rats (weight, $250 \pm 10 \mathrm{~g}$ ) employed in the present study were obtained from the Radiation Study Institute-Animal Center (Tianjin, China). All experimental protocols involving animals were conducted in accordance with guidelines published in the National Institutes of Health Guide for the Care and Use of Laboratory Animals (NIH Publications no. 85-23, revised 1996) and guidelines published by the United Kingdom Coordinating Committee for Cancer Research (18), and were approved by the Ethics Committee of Tianjin Medical University.

The experimental grouping. In the miRNA array experiment, 27 rats were ised. There were 12 rats in the SCI group, 12 rats in the sham group and three rats in control group. The SCI group and sham group included four checkpoints, which in the SCI group were 3, 6, 9 and 12 months following SCI or laminectomy (T1-T4). There were three rats in each checkpoint. The control group was not given any checkpoint. To validate if $\mathrm{Rb} 1$ is the target gene of miR1949, 15 rats were used for immunohistochemisty, with three rats used at each checkpoint (3, 6, 9 and 12 months following SCI) and three rats used as the control group. To investigate the influence of age on the expression levels of miR-1949 and Rb1, nine rats were used for reverse transcription quantitative polymerase chain reaction (RT-qPCR) and western blotting, and a further nine rats were used for immunohistochemisty. In these experiments, there were three checkpoints. Rats in the T1-T3 groups without SCI and laminectomy were grouped together with SCI rats and were sacrificed at an identical as the SCI rats from T2-T4 for sample extraction. To determine the protein expression levels of miR-1949 and Rb1, 12 rats were added, which were used to supply three new checkpoints C1-C3 (1, 14 days and 1 month following SCI) and a control group. There were three rats in the $\mathrm{C} 1-\mathrm{C} 3$ and three rats in the control group. Finally, the protein expression levels of miR-1949 and Rb1 in the C1-C3 and C4-C7 were used togther to determine the protein expression levels of miR-1949 and Rb1 protein.
Surgical methods and sample collection. Forty-five adult female Wistar rats were anesthetized by intraperitoneal injection with $10 \%$ chloral hydrate $(0.3 \mathrm{ml} / 100 \mathrm{~g}$; Tianjin Medical University General Hospital, Tianjin, China). The area to be operated on was shaved and sterilized with $70 \%$ ethanol and betadine (Lierkang, Dezhou, China). The T10 spinous process and vertebral plate were removed, and the dura mater was opened. The exposed spinal cord was struck by a $10 \mathrm{~g}, 25 \mathrm{~mm}$ weight-drop, using a New York University impactor device, as described previously (19). A valid contusive SCI model should comply with the criteria of an SCI model, such that the hindlimbs of the rat twitch involuntarily and the tail moves (20). Sham rats received a laminectomy only. Following surgery, rats were placed in rooms with controlled temperature and humidity, and bladder evacuation was applied daily, prior to the return of reflexive bladder control (21). The rats were sacrificed by intracardial perfusion with $200 \mathrm{ml}$ normal saline (Baiteyiliaoyongping, Shanghai, China).

Following sacrifice of the rats, bladders were excised, and the tissue was either fixed with formalin and embedded in paraffin for immunohistochemistry, or rapidly cut into small sections and snap-frozen in liquid nitrogen, then stored at $-80^{\circ} \mathrm{C}$, for subsequent RNA isolation and western blotting.

RNA isolation. The mirVana ${ }^{\mathrm{TM}}$ RNA Isolation kit (Applied Biosystems, Carlsbad, CA, USA) was used for total RNA isolation. For each tissue mass, 10 volumes of lysis/binding buffer were placed into a plastic tube on ice. Powdered tissue was transferred to the Lysis/Binding buffer, using a pre-chilled metal spatula. The mixture was mixed rapidly and transferred to a vessel. The mixture was then processed to homogeneity using a pulsing vortex mixer (SI-P246; Scientific Industries, New York, NY, USA). miRNA homogenate additive (1/10 volume) was added and chilled for $10 \mathrm{~min}$ on ice. A volume of acid-phenol : chloroform, equal to the lysate volume, was added, prior to adding the miRNA homogenate additive and mixing via vortex for 30-60 sec. In order to separate the organic and aqueous phases, centrifugation was performed for $5 \mathrm{~min}$ at room temperature, at the maximum speed $(10,000 \mathrm{xg})$ and the aqueous phase was transferred to a fresh tube without disturbing the organic phase. The volume removed was recorded, and 1.25 volume $100 \%$ ethanol was added and mixed thoroughly. A filter cartridge was put into one of the collection tubes supplied for each sample. The lysate/ethanol mixture was applied onto the filter cartridge using a pipette. The mixture was centrifuged for $15 \mathrm{sec}$ at $10,000 \mathrm{x} \mathrm{g}$, in order to pass it through the filter. Alternatively, vacuum pressure may be used to pass samples through the filter. This process was repeated until the entire lysate/ethanol mixture had passed through the filter and the flow-through was discarded. The collection tube was reused for the washing steps. The filter was washed with $700 \mu \mathrm{l}$ miRNA wash solution 1 and then washed twice with $500 \mu \mathrm{l}$ wash solution $2 / 3$. Elution solution $(100 \mu \mathrm{l})$ that had been preheated to $95^{\circ} \mathrm{C}$, was applied to the center of the filter, which was covered with the cap. Samples were spun for 20-30 sec at 10,000 x $\mathrm{g}$ in order to recover the eluate, which contained the RNA, and were stored at $-70^{\circ} \mathrm{C}$.

miRNA array and data analysis. In order to assess the differential expression of miRNAs, 27 samples were used from the T1-T4 time points $(3,6,9$ and 12 months following SCI or laminectomy). Four SCI groups and four sham groups from each of the 
A

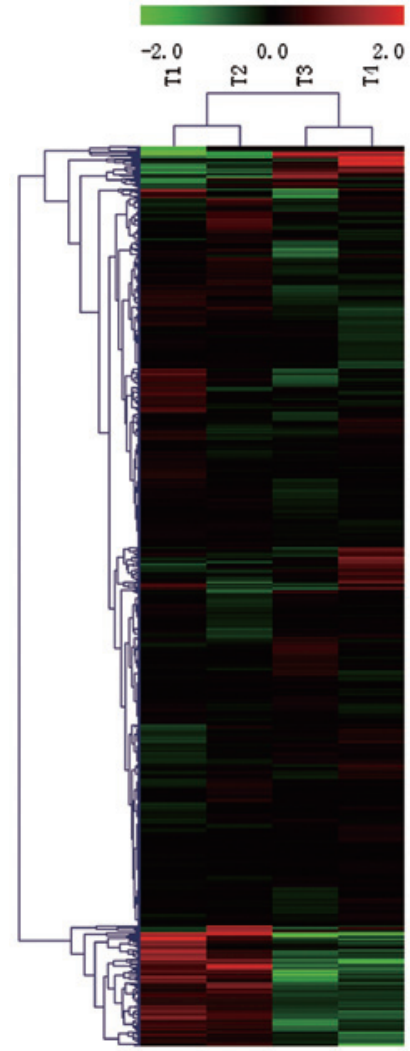

B

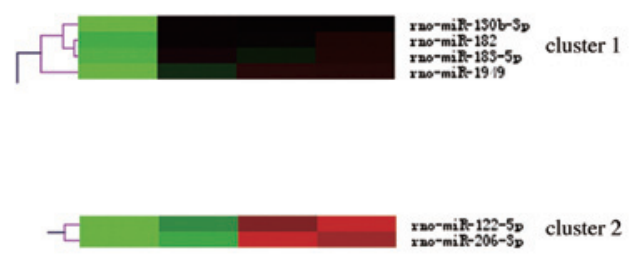

$-$
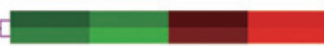

$\underset{\substack{x=0-m i R-150-S_{D} \\ \text { xao-miR }-652-5_{p}}}{\text { cluster } 3}$
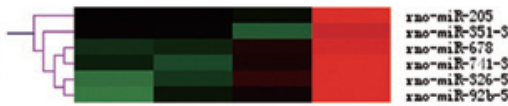

cluster 4

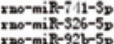
cluster 4
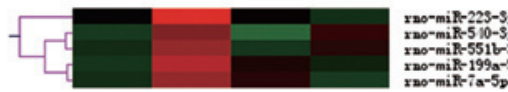

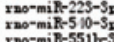

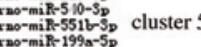

Figure 1. Expression profiles of miRNAs in rat bladder following SCI. (A) Heat map of microarray expression data from four time points (T1-T4). The expression of miRNAs, analyzed by Hierarchical Clustering, is shown on the y-axis, and the four time points, analyzed by Hierarchical Clustering, are shown on the $\mathrm{x}$-axis. The relative miRNA expression is depicted according to the color scale above. Red indicates upregulation; green, downregulation. (B) miRNAs is which the RMA normalization signal value of the experimental group was $\geq 5$, the RMA normalization signal value ratio of experimental group to that of the sham group was $\geq 2$, and the fold change was $\geq 4$ ), belong to these five clusters. T1-T4 = 3, 6,9 and 12 months after SCI. miRNA, microRNA; SCI, spinal cord injury; RMA, robust multi-array average.

four time points (T1-T4), and one control group (rats without SCI and laminectomy), were included. Each group contained three samples. The NanoDrop ND-2100 (Thermo Fisher Scientific, Waltham, MA, USA) was used to quantify total RNA, and the Agilent 2100 (Agilent Technologies, Inc., Santa Clara, CA, USA) was used to assess RNA integrity. Labeling, hybridization and washing were performed according to the manufacturer's instructions. Briefly, Poly A was tailed to total RNA, which was then labeled by Biotin. After they were labeled, RNAs were hybridized onto the microarray (Affymetrix 902017, Affymetrix Santa Clara, USA). Following washing and staining, scanning was performed with the Affymetrix Scanner 3000 (Affymetrix, Inc., Santa Clara, CA, USA).

Expression Console software (version1.3.1, Affymetrix, Inc.) was used to analyze array images in order to extract raw data and provide robust multi-array average (RMA) normalization. The resulting data was processed by Genespring software (version 12.5; Agilent Technologies, Inc.). Differential expression of miRNAs was then identified by evaluation of fold changes. The Targetscan database (http://www.targetscan.org) was used to predict the target genes of differentially expressed miRNAs. The distinguishable expression pattern of miRNAs among samples was demonstrated by performing Hierarchical Clustering using Multi Experiment Viewer 4.9 software.

$R T$-qPCR. Quantification was performed with a two-step reaction proces, RT and PCR. Each RT reaction consisted of $0.5 \mu \mathrm{g}$ RNA, $2 \mu \mathrm{l}$ PrimerScript Buffer, $0.5 \mu \mathrm{l}$ oligo dT, $0.5 \mu \mathrm{l}$ random 6mers and $0.5 \mu$ l PrimerScript RT Enzyme Mix I (Takara, Otsu, Japan), in a total volume of $10 \mu \mathrm{l}$. The reactions were performed in a GeneAmp® PCR System 9700 (Applied Biosystems) for $15 \mathrm{~min}$ at $37^{\circ} \mathrm{C}$, followed by heat inactivation of RT for $5 \mathrm{sec}$ at $85^{\circ} \mathrm{C}$. For miRNA, each RT reaction consisted of $1 \mu \mathrm{g}$ RNA, $4 \mu 1$ miScript HiSpec Buffer, $2 \mu \mathrm{l}$ Nucleics mix and $2 \mu \mathrm{l}$ miScript Reverse Transcriptase mix (Qiagen, Hilden, Germany) in a total volume of $20 \mu \mathrm{l}$. Reactions were performed in a GeneAmp ${ }^{\circledR}$ PCR System 9700 (Applied Biosystems, USA) for $60 \mathrm{~min}$ at $37^{\circ} \mathrm{C}$, followed by heat inactivation of RT for $5 \mathrm{~min}$ at $95^{\circ} \mathrm{C}$. For mRNA, the expression of miRNAs and mRNA was normalized to that of U6 or GADPH, respectively. Each sample was performed in triplicate. The primer sequences (Oebiotech, Shanghai, China) were as follows: U6, 5'-CAAGGATGACACGCAAATTCG-3'; rno-miR-1949, 5'-TATACCAGGATGTCAGCATAGTT-3'; rno-miR-205, 5'-TCCTTCATTCCACCGGAGTCTGT-3'; rnomiR-150-3p, forward 5'-ATTTACTGGTACAGGCCTGGG-3' and reverse 5'-CAGTGCAGGGTCCGAGGTAT-3'; rno-miR652-5p, forward 5'-AAGGACAACCCTAGGAGGGG-3' and reverse 5'-CGCAGGGTCCGAGGTATTC-3'; Rb1, forward 5'-TGTGATGTTTGCTCTTGGT-3' and reverse 5'-GAATGTGGACAATCAATCAACT-3'; GAPDH, forward 5'-GCGAGATCCCGCTAACATCA-3' and reverse 5'-CTCGTGGTTCACACCCATCA-3'.

Western blotting. In order to investigate the expression of the Rb1 protein, western blotting was performed. Briefly, bladder samples of rats were lysed in RIPA buffer (Santa Cruz 
Biotechnology, Inc., Dallas, TX, USA). Following supplementation with protease inhibitor cocktail (Sigma-Aldrich, St. Louis, MO,USA), the lysates were centrifuged in order to isolate protein. Protein samples were separated using SDS-polyacrylamide gel electrophoresis (Tuheshiye, Shanghai, China) and then transferred to nitrocellulose membranes (Tuheshiye). Membranes were blocked with 5\% non-fat milk in PBS, containing $0.05 \%$ Tween-20, in order to reduce non-specific antibody binding. Blots were then incubated with rabbit anti-human $\mathrm{Rb}$ antibody primary antibodies (1:500; cat. no. GTX50459; GeneTex). $\beta$-actin was used as internal control. A got anti-rabbit immunoglobulain $\mathrm{G}$ horseradish perxoidase linked secondary antibody (1:2,000; cat. no. HA1001; Huaan, Hangzhou, China) was used to detect bound primary antibody. All experiments were conducted in triplicate.

Immunohistochemistry. In order to demonstrate the presence of the Rb1 protein, immunohistochemistry was used. In brief, formalin-fixed and paraffin-embedded samples were cut into sections. The histological sections were deparaffinised with xylene, rehydrated through graded ethanol solutions and washed in distilled water. After endogenous peroxidase activity had been blocked using $0.3 \%$ hydrogen peroxide for $15 \mathrm{~min}$, the sections were submitted to antigen retrieval by microwaving 3 times for 5 min each and then cooling to room temperature. Following washing with distilled water and TBS, the primary antibody to $\mathrm{Rb}$ was added at room temperature in a wet chamber. Following thorough washing with TBS, the sections were incubated with the rabbit secondary antibody (ImmunoCruz ${ }^{\mathrm{TM}} \mathrm{ABC}$ staining system; 1:500; cat. no. sc-2018; Santa Cruz Biotechnology, Dallas, USA) in a wet chamber at room temperature and stained with DAB (Saichi, Beijing, China). The sections were then counter-stained with hematoxylin.

Statistical analysis. Data are reported as the mean \pm standard deviation. Comparisons between two groups were analyzed using Student's $t$-test using SPSS 18.0 software (SPSS, Inc., Chicago, IL, USA). $\mathrm{P}<0.05$ was considered to indicate a statistically significant difference.

\section{Results}

Expression profiles of miRNAs in the rat bladder following $S C I$. Following array processing, Expression Console software (version1.3.1, Affymetrix, Inc.) was used to analyze array images in order to obtain raw data and then to provide RMA normalization. Based on the literature pertaining to the duration of SCI at the time of diagnosis of bladder cancer, and using a formula to adjust these times to the lifespan of a rat, miRNA expression data were collected from rat bladders at 3,6,9 and 12 months following SCI (time points, T1-T4) $(8,22,23)$.

Microarray analysis demonstrated that 381 miRNAs were differentially expressed in the adult rat bladder following SCI. Hierarchical Clustering was performed in order to illustrate the expression pattern of the distinguishable miRNAs among the samples (Fig. 1A).

Since only a small proportion of miRNAs are abundantly expressed in the microRNAome, and as they seem to be more important in bladder biology and carcinogenic effects, only those miRNAs which were fulfilled following criteria that the

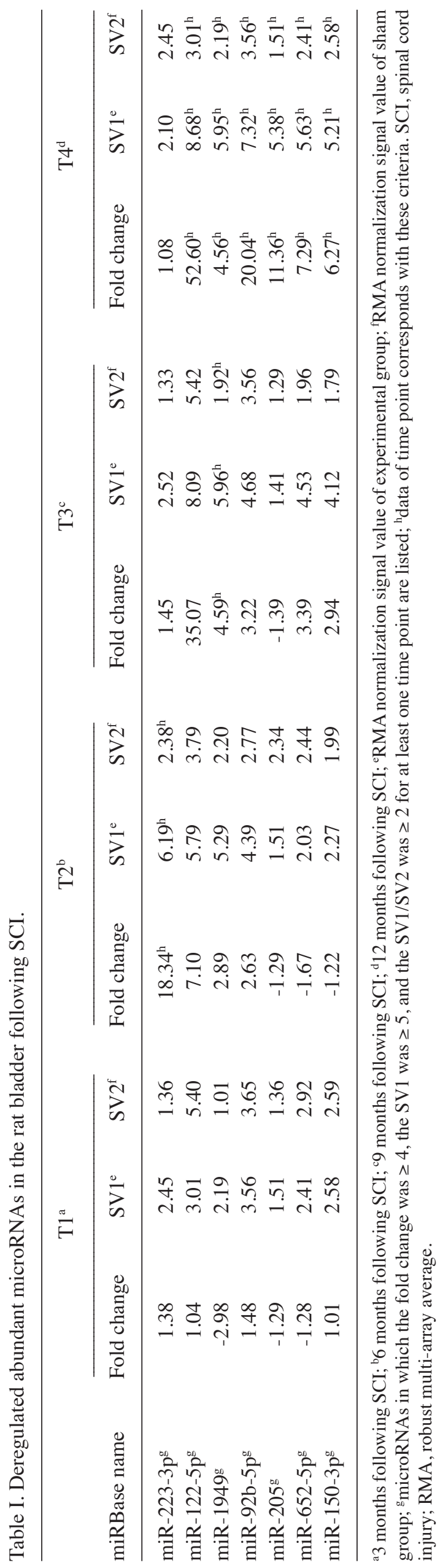


A

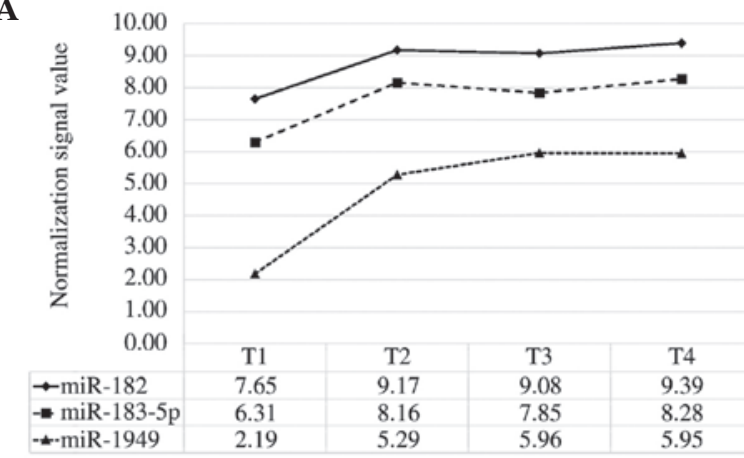

B

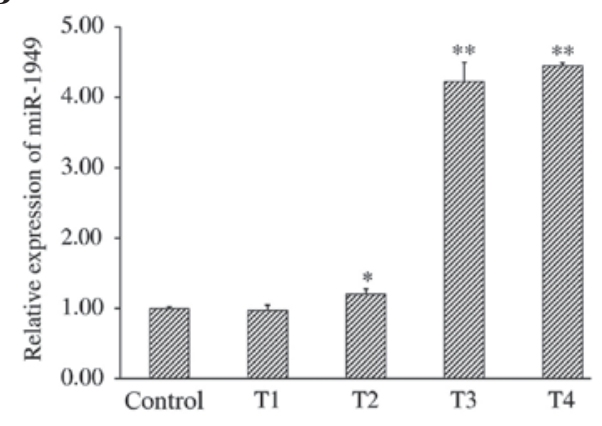

C

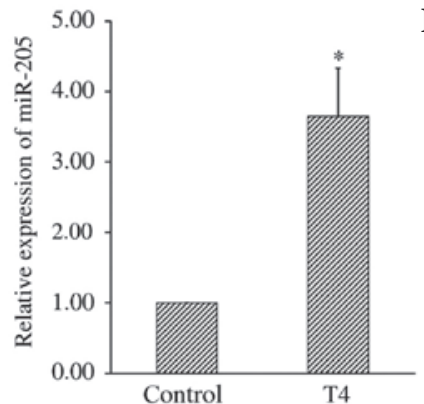

D

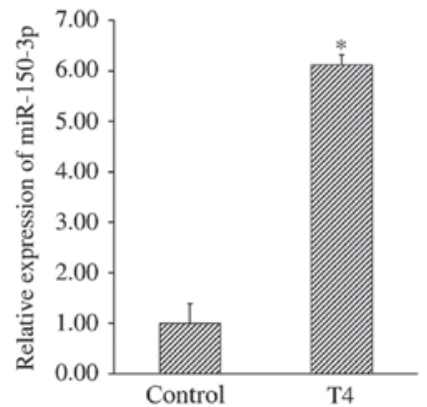

$\mathbf{E}$

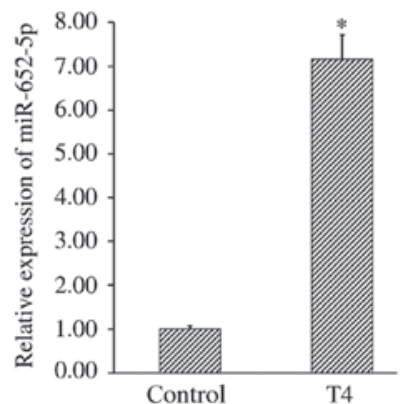

Figure 2. Expression trends of miR-182, miR-183-5p and miR-1949, and validation of microarray data by qRT-PCR. (A) Expression trends of miR-182, miR-183-5p and miR-1949 over four time points (T1-T4) were analyzed by microarray. (B) The relative expression of miR-1949 at four time points (T1-T4) was measured by qRT-PCR and normalized to that of the internal control (U6). (C), (D) and (E) The relative expression of miR-205, miR-150-3p and miR-652-5p at the check point for which the RMA normalization signal value of the experimental group was $\geq 5$, the RMA normalization signal value ratio of the experimental group to that of the sham group was $\geq 2$, and the fold change $\geq 4$ ) was analyzed by qRT-PCR and normalized to the internal control (U6). "P $<0.05$ and ${ }^{* *} \mathrm{P}<0.005$, compared with the control group). T1-T4=3, 6, 9 and 12 months following spinal cord injury. miRNA, microRNA; qRT-PCT, quantitative reverse transcription-polymerase chain reaction; RMA, robust multi-array average.

RMA normalization signal value was $\geq 5$ at one time point, the ratio of the RMA normalization signal value of the experimental group to that of the sham group was $\geq 2$, and the fold change was $\geq 4$, were considered likely to be involved in bladder cancer pathogenesis $(3,4,14)$. Based on these criteria, seven miRNAs were identified, which are shown in Table I.

Deregulated abundant miRNAs in the rat bladder following SCI. A more thorough investigation of the miRNAs in Table I was conducted in order to identify the potential mechanisms underlying the development of bladder cancer, and the miRNAs responsible, by examining numerous published studies. To the best of our knowledge, there is no evidence suggesting that six of the seven miRNAs (miR-223-3p, miR-122-5p, miR-1949, miR-92b-5p, miR-652-5p and miR-150-3p) are associated with the development of bladder cancer. A number of studies have reported that miR-205 is a biomarker for the diagnosis, staging and prognosis of bladder cancer. However, it has not been reported that miR-205 or its target genes induce bladder cancer (24-31).

Subsequently, clusters of the miRNAs in Table I were investigated, according to the hypothesis that miRNAs in the same cluster, as analyzed by Hierarchical Clustering, may perform similar biological functions and exhibit similar expression patterns. The seven miRNAs in Table I belonged to five clusters (Fig. 1B).

To the best of our knowledge, there are no previous reports that miRNAs in three of these clusters (2, 3 and 5) are associated with bladder cancer. Among the miRNAs in cluster 4, none are associated with bladder cancer, with the exception of miR-205. miR-205, a biomarker for the diagnosis, grading and prognosis of bladder cancer, is not associated with the biological mechanisms underlying tumorigenesis in the bladder (24-31).

Previous reports have shown that all members of cluster 1 , with the exception of miR-1949, that is, miR-130b-3p, miR-182 and miR-183-5p, are biomarkers of bladder cancer, and their upregulation was also observed in the present study (32-37). As shown in Fig. 2A, the change in expression of miR-1949 over time, was similar to that of miR-182 and miR-183-5p. miR-182 and miR-183-5p are the most frequently reported deregulated miRNAs in studies of bladder cancer studies. However, the increase in their expression was not as marked as that of miR-1949. Therefore, miR-1949 was further investigated.

$q R T-P C R$ validates the profiling data. In order to confirm the accuracy of the microarray data, qRT-PCR was used to measure the expression of miR-1949 at four time points (T1-T4), and that of miR-205, miR-652-5p and miR-150-3p at the time point for which the change in their expression, as assessed by the microarray, was consistent with the criteria already described. The correlation between microarray and qRT-PCR data was strong for these miRNAs, with the exception of miR-205. The fold change of miR-205 according to qRT-PCR, was 3.65, which was not consistent with the microarray data, although a statistical difference still existed, compared withe the control group. The relative expression of each miRNA compared with that in control subjects is shown in Fig. 2B-E.

miR-1949 targets $R b 1$. As miRNA functions primarily by inhibiting the mRNA of target genes, the target of miR-1949 that is known to be involved in the pathogenesis of bladder cancer was 
A

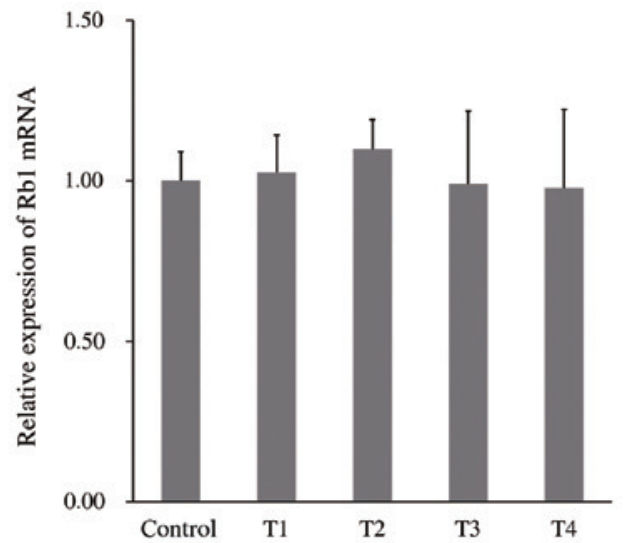

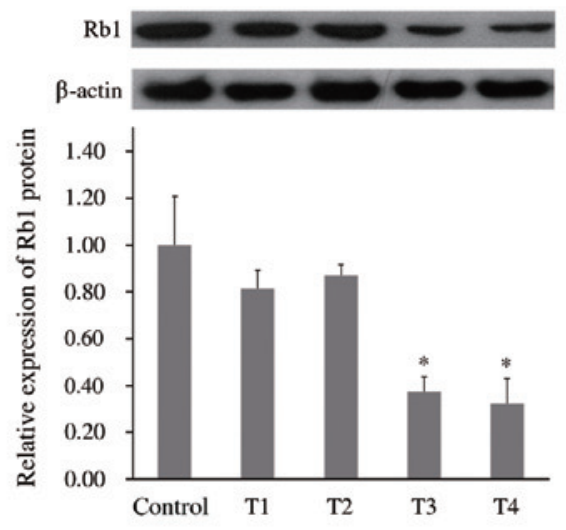

C

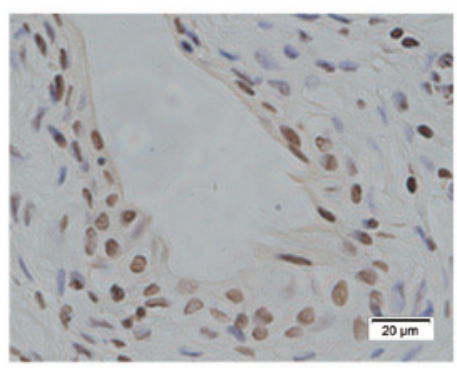

D

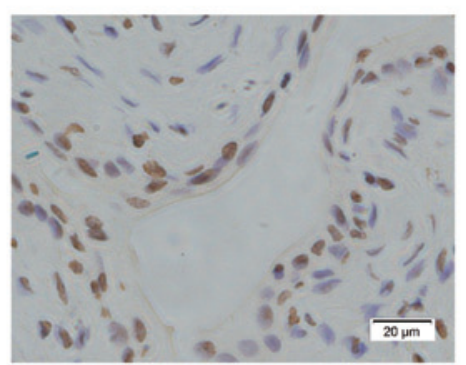

E

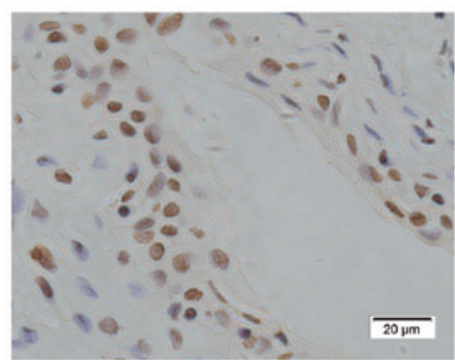

F

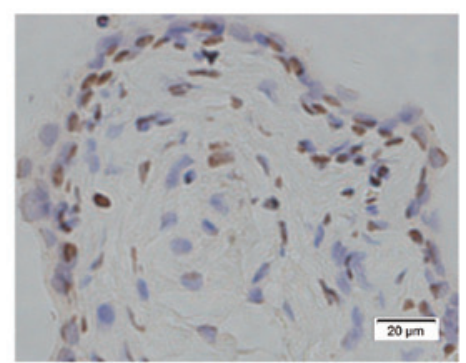

G

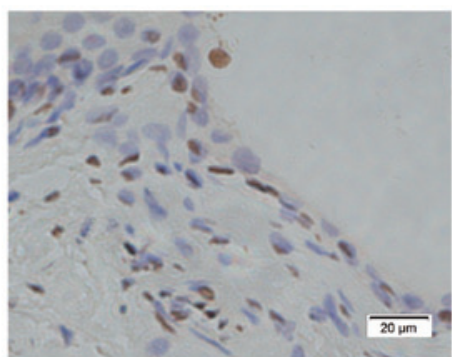

Figure 3. miR-1949 targets Rb1. (A) Relative expression of Rb1 mRNA at four time points (T1-T4) was measured using qRT-PCR and normalized to the expression of the internal control (GADPH). (B) The protein level of Rb1 at four time points (T1-T4) was analyzed by western blotting and normalized to the level of $\beta$-actin. Rb1 protein levels significantly decreased at T3 and T4. "P<0.05, compared with the control group. (C), (D), (E), (F) and (G) Immunohistochemical staining showing bladder tissue Rb1 protein from the control group and T1-T4 time points, respectively. Magnification, $\mathrm{x} 400$. T1-T4=3, 6, 9 and 12 months following spinal cord injury. Rb1, retinoblastoma 1; qRT-PCR, quantitative reverse transcription-polymerase chain reaction.

further analyzed. The target genes of miR-1949 were computationally predicted by TargetScan. Computational prediction indicated that miR-1949 may target Rb1. It has been shown that the absence of the Rb1 protein is involved in several pathways in bladder cancer, either at its initiation or during its progression, including cell-cycle regulation and apoptosis $(16,17)$. It is hypothesized that the Rb1 gene is involved in bladder tumori In order to assess the differential expression of miRNAs, 27 samples were used from the T1-T4 time points (3, 6, 9 and 12 months following SCI or laminectomy). Four SCI groups and four sham groups from each of the four time points (T1-T4), and one control group (rats without SCI and laminectomy), were included genesis, as tumorigenicity may be suppressed by transfecting the Rb1 gene into bladder cancer cells (16).

In order to investigate the regulation of Rb1 by miR-1949, the expression of Rb1 mRNA was measured using qRT-PCR. The relative expression of Rb1 at four time points (T1-T4) compared with that in normal control subjects is shown in Fig. 3A. The level of Rb1 mRNA was not significantly influenced by the expression of miR-1949. Further, western blotting of four time points (T1-T4) samples demonstrated that the level of the Rb1 protein was decreased at T3 and T4 (Fig. 3B), which was further confirmed by immunohistochemistry (Fig. 3C-G). This suggested that Rb1 expression may be inhibited by miR-1949, primarily through translational inhibition. miRNA has two modalities to inhibit it target proteins: One is to inhibit its target mRNA and another is to degrade its target mRNA, if the miRNA is high compared with its target mRNA (1-3). In the first modality, the level of target mRNA remains unchanged. These data indicate that endogenous Rb1 is targeted and regulated by miR-1949, and also suggest that the potential carcinogenic effect of miR-1949 is due to the inhibition of Rb1.

Influence of age on miR-1949 and Rbl protein. As tumorigenesis and the expression of certain miRNAs has been shown to be associated with aging (38-40), the levels of miR-1949 and Rb1 protein were measured in rats of different ages. The healthy rats from the control group and the t1-t3 groups without SCI and laminectomy, which were employed together with SCI rats, were sacrificed at the same time as the SCI rats from the T1-T4 groups, in order 
A

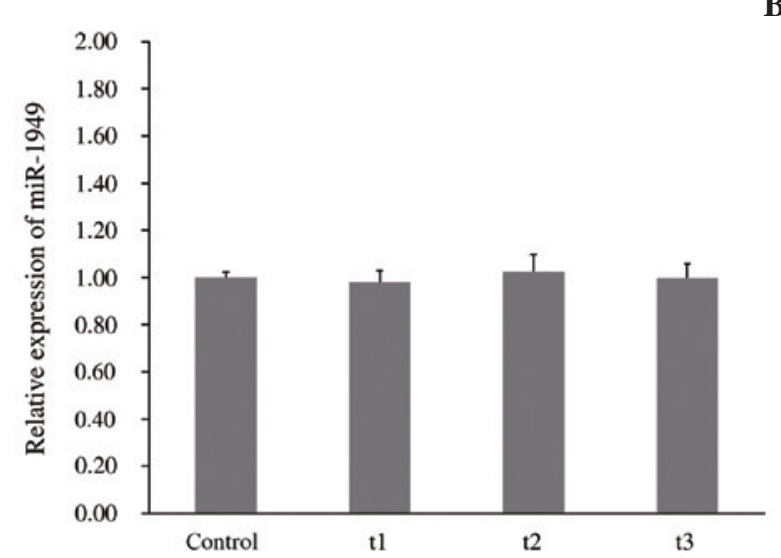

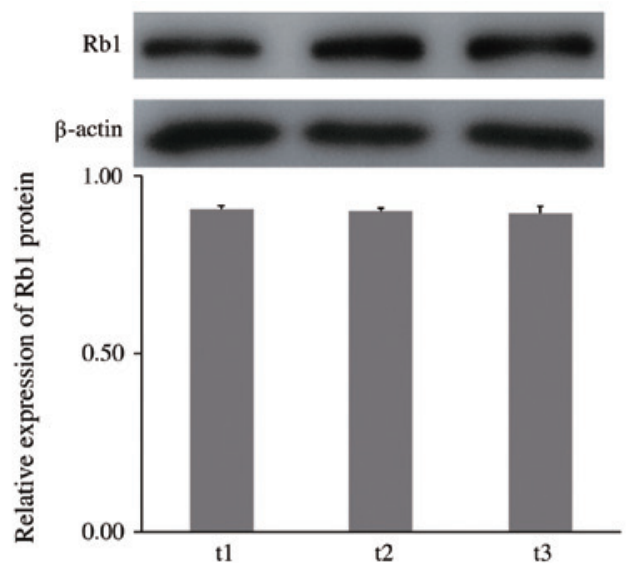

C

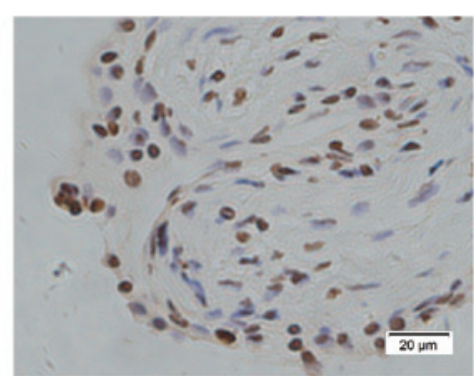

D

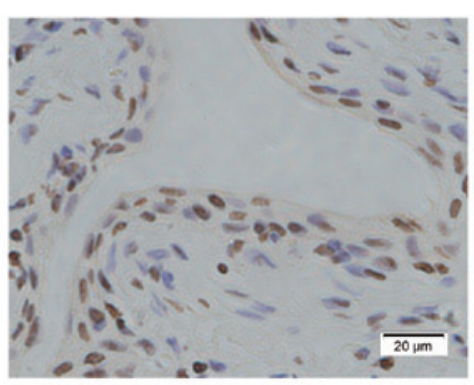

$\mathbf{E}$

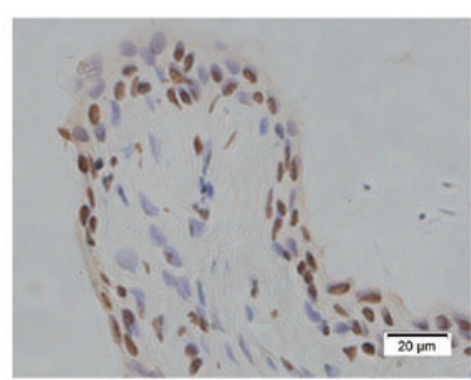

Figure 4. Influence of age on miR-1949 and Rb1 protein. (A) Relative expression of miR-1949 was analyzed by qRT-PCR and normalized to the internal control (U6). (B) The protein level of Rb1 was analyzed by western blotting and normalized to $\beta$-actin. (C), (D) and (E) Immunohistochemical staining showing bladder tissue Rb1 protein at t1, t2 and t3, respectively. Magnification, x400. Rats of the t1-t3 groups without SCI and laminectomy, which were used together with SCI rats, were sacrificed at an identical time to SCI rats from T2-T4, to extract samples. T1-T4=3, 6, 9 and 12 months following SCI. SCI, spinal cord injury; Rb1, retinoblastoma 1; qRT-PCR, quantitative reverse transcription-polymerase chain reaction.

A

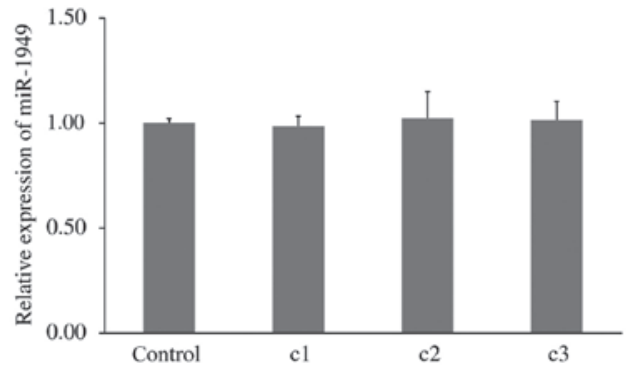

C

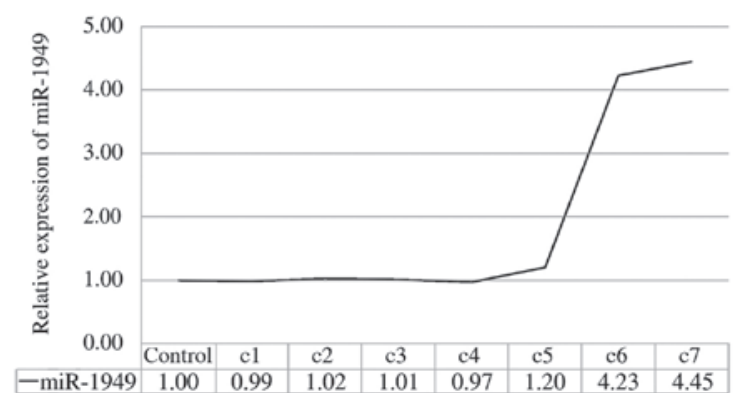

B

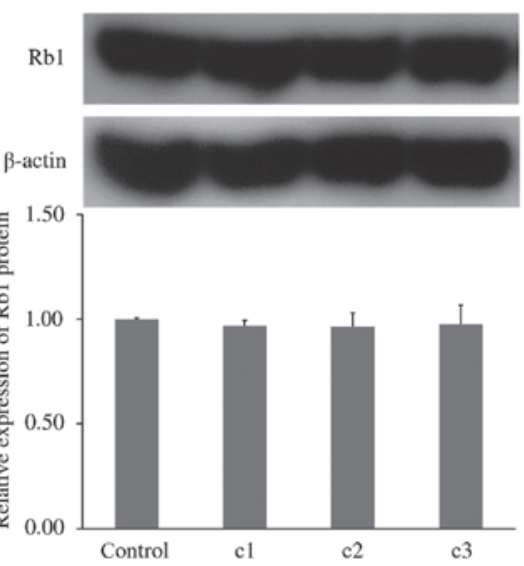

D

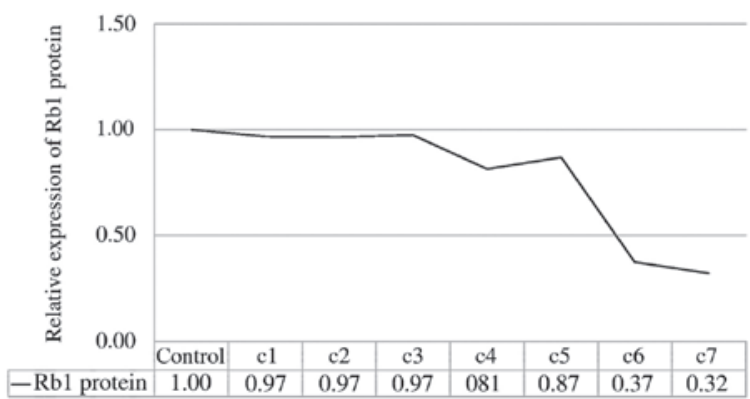

Figure 5. Overall trends of miR-1949 and the Rb1 protein. (A) Expression level of miR-1949 in rat bladders was measured by qRT-PCR at 1 day, 14 days and 1 month following SCI. (B) Protein level of Rb1 at 1 day, 14 days and 1 month following SCI. (C) Overall trend of miR-1949, which was stable until c4, and then increased significantly. (D) Overall trend of the Rb1 protein. It decreased significantly at c5. c1-7=1 day, 14 days, and 1, 3, 6, 9 and 12 months following SCI. Rb1, retinoblastoma 1; qRT-PCR, quantitative reverse transcription-polymerase chain reaction; SCI, spinal cord injury. 
to extract samples for qRT-PCR. Each group included three rats. No significant differences were detected, which suggested that miR-1949 levels are not influenced by age (Fig. 4A). The Rb1 protein levels were analyzed by western blotting at different time points (t1-t3), and no significant difference was detected among these groups, indicating that the level of the Rb1 protein is also not influenced by age (Fig. 4B). Furthermore, this result was validated by immunohistochemistry (Fig. 4C-E).

Overall trends of miR-1949 and Rbl protein expression. The expression levels of miR-1949 and Rb1 protein in rat bladders were measured by qRT-PCR and western blotting, respectively, at 1 day, 14 days and 1 month following SCI, and no significant difference was detected among the groups (Fig. 5A and B). In order to analyze the overall trends of the expression of miR-1949 and Rb1 protein, the data from the T1-T4 time points were used and are indicated as c4-c7 in Fig. 5C and D, respectively. The expression of miR-1949 remained stable for 3 months following SCI, and then significantly increased, a trend that was maintained for at least 9 months (Fig. 5C). The expression of the $\mathrm{Rb} 1$ protein did not significantly decrease until c5 (Fig. 5D). If these trends are maintained, the Rb1 protein levels may decline to a carcinogenic level.

\section{Discussion}

miRNAs are small non-coding RNA molecules that regulate gene expression by interacting with target mRNAs. These post-transcriptional regulators serve to maintain the characteristic protein expression of distinct cellular phenotypes $(2,41)$. Although translation is blocked, the target mRNAs are thought to be degraded only by those miRNAs with high complementarity $(1,3)$. The level of Rb1 mRNA was not significantly influenced by miR-1949 expression, suggesting that Rb1 expression may be inhibited by miR-1949 primarily through translational inhibition.

When miRNAs were identified, they were identified as a class of gene regulators, which were associated with cell growth, differentiation and apoptosis. Notably, deregulation of these miRNAs may cause them to function as 'oncomiRs', which induce tumorigenesis and promote tumor development. Therefore, knowledge of the tissue microRNAome as a potential microenvironment for inducing tumorigenesis is a prerequisite for an improved understanding of cancer cell biology, and also extends the number of existing molecules that may be developed for use as accurate diagnostic makers and efficient therapeutic targets (4).

Microarray is a powerful high-throughput tool which is able to monitor the global miRNA expression dynamically within tens of samples that are processed in parallel during a single experiment (42). Compared with investigation of the expression of single miRNAs, microarray technology makes it possible to study the mechanism of disease and injury in a systemic and global context.

It has been reported that miRNAs that are expressed at a low level are unlikely to perform biological functions, and a minimum expression threshold amount must be achieved in order to inhibit target mRNAs $(4,43,44)$, suggesting that the intensity expression values of miRNAs in the bladder microRNAome have more possibility to execute their biological functions (3). In the candidate lists obtained in the present study, miRNAs were prioritized if they exhibited an RMA normalization signal value in the experimental group of $\geq 5$, in order to identify the miRNAs with potential biological function.

The absence of the Rb1 protein is involved in a number of pathways in bladder cancer, either at its initiation or during its progression $(16,17)$. Alteration or deletion of the Rb1 protein is observed in human bladder cancer, and transfecting the Rb1 gene into bladder cancer cells suppresses the tumorigenicity of these cells (16). The alteration in Rb1 protein expression may independently predict recurrence and progression in bladder cancer with Bacillus Calmette-Guerin administration, and low expression of $\mathrm{Rbl}$ is a predictor of non-response and cancer recurrence $(45,46)$.

One of the first studies to show an increased risk of bladder cancer following SCI was performed by Kawaichi (47). A number of studies have demonstrated that independent risk factors for the development of bladder cancer in patients with SCI, include age, long-term indwelling catheters, recurrent UTIs and bladder stones $(12,48,49)$. However, none of these studies has explored the pathogenesis of bladder cancer following SCI at the miRNA level. To the best of our knowledge, the present study is the first to explore the potential mechanism of the increased incidence of bladder cancer following SCI in the context of the microRNAome.

Based on the literature pertaining to the duration of SCI at the time of diagnosis of bladder cancer, and using a formula to adjust these times to the lifespan of a rat, four time points (3, 6,9 and 12 months following SCI) were selected for use in the present study $(8,22,23)$. Since the pathological environment of the bladder following SCI differ from the normal bladder, investigation of the potential association between miRNA and the protein expression of Rb1 with the occurrence of bladder cancer following SCI, is required.

However, as a high number of experimental animals are required to obtain bladder cancer cases from rats with SCI, and the lifespan of experimental animals is limited, the present study is restricted to potential underlying mechanisms and the identification of miRNAs that may be involved in this process $(8,10,22,23)$. However, a long-term clinical study with a large sample is also necessary.

In conclusion, the present study suggests that miR-1949 is involved in the translational regulation of $\mathrm{Rbl}$, and also that the upregulation of miR-1949 may promote tumorigenesis via targeting Rb1. Future clinical studies aiming at understanding how miRNAs contribute to the development of bladder cancer following SCI, may help to further elucidate the role of these small non-coding RNAs.

\section{Acknowledgements}

This study was supported by the Medical Science and Technology Youth Cultivation Project of the Chinese People's Liberation Army (grant no. 13QNP017), the Key Program of National Natural Science Foundation of China (grant no. 81330042), the General Program of National Natural Science Foundation of China (grant nos. 81371957 and 81171714), the Special Project between Ministry of Science and Technology of China and Russia (grant no. 2014DFR31210) and the Cooperation Project between Tianjin Municipal Science and Technology Commission and Australia (grant no. 13RCGFSY19000). 


\section{References}

1. Williams AE: Functional aspects of animal microRNAs. Cell Mol Life Sci 65: 545-562, 2008.

2. Nagalla S, Shaw C, Kong X, et al: Platelet microRNA-mRNA coexpression profiles correlate with platelet reactivity. Blood 117: 5189-5197, 2011.

3. Hou J, Lin L, Zhou W, et al: Identification of miRNomes in human liver and hepatocellular carcinoma reveals miR-199a/b-3p as therapeutic target for hepatocellular carcinoma. Cancer Cell 19: 232-243, 2011.

4. Munding JB, Adai AT, Maghnouj A, et al: Global microRNA expression profiling of microdissected tissues identifies miR-135b as a novel biomarker for pancreatic ductal adenocarcinoma. Int J Cancer 131: E86-E95, 2012.

5. Hua R, Shi J, Wang X, et al: Analysis of the causes and types of traumatic spinal cord injury based on 561 cases in China from 2001 to 2010. Spinal cord 51: 218-221, 2013.

6. Bejany DE, Lockhart JL and Rhamy RK: Malignant vesical tumors following spinal cord injury. J Urol 138: 1390-1392, 1987.

7. Groah SL, Weitzenkamp DA, Lammertse DP, Whiteneck GG Lezotte DC and Hamman RF: Excess risk of bladder cancer in spinal cord injury: Evidence for an association between indwelling catheter use and bladder cancer. Arch Phys Med Rehabil 83: 346-351, 2002.

8. Subramonian K, Cartwright RA, Harnden P and Harrison SC: Bladder cancer in patients with spinal cord injuries. BJU Int 93: 739-743, 2004.

9. Kalisvaart JF, Katsumi HK, Ronningen LD and Hovey RM: Bladder cancer in spinal cord injury patients. Spinal Cord 48: 257-261, 2010

10. Kaufman JM, Fam B, Jacobs SC, et al: Bladder cancer and squamous metaplasia in spinal cord injury patients. J Urol 118 967-971, 1977.

11. Geisse LJ and Tweeddale DN: Pre-clinical cytological diagnosis of bladder cancer. J Urol 120: 51-56, 1978.

12. Pannek J: Transitional cell carcinoma in patients with spinal cord injury: A high risk malignancy? Urology 59: 240-244, 2002

13. West DA, Cummings JM, Longo WE, Virgo KS, Johnson FE and Parra RO: Role of chronic catheterization in the development of bladder cancer in patients with spinal cord injury. Urology 53: 292-297, 1999.

14. Lau P, Verrier JD, Nielsen JA, Johnson KR, Notterpek L and Hudson LD: Identification of dynamically regulated microRNA and mRNA networks in developing oligodendrocytes. J Neurosci 28: 11720-11730, 2008

15. Sparkes RS, Sparkes MC, Wilson MG, et al: Regional assignment of genes for human esterase D and retinoblastoma to chromosome band 13q14. Science 208: 1042-1044, 1980.

16. Miyamoto H, Shuin T, Torigoe S, Iwasaki Y and Kubota Y: Retinoblastoma gene mutations in primary human bladder cancer. Br J Cancer 71: 831-835, 1995.

17. Mitra AP, Hansel DE and Cote RJ: Prognostic value of cell-cycle regulation biomarkers in bladder cancer. Semin Oncol 39 524-533, 2012

18. Workman P, Aboagye EO, Balkwill F, et al: Guidelines for the welfare and use of animals in cancer research. Br J Cancer 102: $1555-1577,2010$

19. Ban DX, Kong XH, Feng SQ, Ning GZ, Chen JT and Guo SF Intraspinal cord graft of autologous activated Schwann cells efficiently promotes axonal regeneration and functional recovery after rat's spinal cord injury. Brain Res 1256: 149-161, 2009.

20. Zhou HX, Li XY, Li FY, et al: Targeting RPTPsigma with lentiviral shRNA promotes neurites outgrowth of cortical neurons and improves functional recovery in a rat spinal cord contusion model. Brain research 1586: 46-63, 2014.

21. Liu Y, Wang CY, Kong XH, et al: Novel multifunctional polyethylene glycol-transactivating-transduction protein-modified liposomes cross the blood-spinal cord barrier after spinal cord injury. J Drug Target 18: 420-429, 2010.

22. Andreollo NA, Santos EF, Araújo MR and Lopes LR: Rat's age versus human's age: What is the relationship? Arq Bras Cir Dig 25: 49-51, 2012 (In English, Portuguese).

23. Quinn R: Comparing rat's to human's age: How old is my rat in people years? Nutrition 21: 775-777, 2005.

24. Lee H, Jun SY, Lee YS, Lee HJ, Lee WS and Park CS: Expression of miRNAs and ZEB1 and ZEB2 correlates with histopathological grade in papillary urothelial tumors of the urinary bladder. Virchows Arch 464: 213-220, 2014.

25. Ratert N, Meyer HA, Jung M, et al: miRNA profiling identifies candidate mirnas for bladder cancer diagnosis and clinical outcome. J Mol Diagn 15: 695-705, 2013.
26. Tran MN, Choi W, Wszolek MF, et al: The p63 protein isoform $\Delta$ Np63 $\alpha$ inhibits epithelial-mesenchymal transition in human bladder cancer cells: Role of MIR-205. J Biol Chem 288: 3275-3288, 2013

27. Dip N, Reis ST, Timoszczuk LS, et al: Stage, grade and behavior of bladder urothelial carcinoma defined by the microRNA expression profile. J Urol 188: 1951-1956, 2012.

28. Wang G, Chan ES, Kwan BC, et al: Expression of microRNAs in the urine of patients with bladder cancer. Clin Genitourin Cancer 10: 106-113, 2012.

29. Wiklund ED, Bramsen JB, Hulf T, et al: Coordinated epigenetic repression of the miR-200 family and miR-205 in invasive bladder cancer. Int J Cancer 128: 1327-1334, 2011.

30. Neely LA, Rieger-Christ KM, Neto BS, et al: A microRNA expression ratio defining the invasive phenotype in bladder tumors. Urol Oncol 28: 39-48, 2010.

31. Gottardo F, Liu CG, Ferracin M, et al: Micro-RNA profiling in kidney and bladder cancers. Urol Oncol 25: 387-392, 2007.

32. Friedman JM, Liang G, Liu CC, et al: The putative tumor suppressor microRNA-101 modulates the cancer epigenome by repressing the polycomb group protein EZH2. Cancer Res 69: 2623-2629, 2009.

33. Han Y, Chen J, Zhao X, et al: MicroRNA expression signatures of bladder cancer revealed by deep sequencing. PLoS One 6: e18286, 2011.

34. Yamada Y, Enokida H, Kojima S, et al: MiR-96 and miR-183 detection in urine serve as potential tumor markers of urothelial carcinoma: Correlation with stage and grade, and comparison with urinary cytology. Cancer Sci 102: 522-529, 2011.

35. Pignot G, Cizeron-Clairac G, Vacher S, et al: microRNA expression profile in a large series of bladder tumors: Identification of a 3-miRNA signature associated with aggressiveness of muscle-invasive bladder cancer. Int J Cancer 132 2479-2491, 2013

36. Yoshino H, Seki N, Itesako T, Chiyomaru T, Nakagawa M and Enokida $\mathrm{H}$ : Aberrant expression of microRNAs in bladder cancer. Nat Rev Urol 10: 396-404, 2013.

37. Zhang QH, Sun HM, Zheng RZ, et al: Meta-analysis of microRNA-183 family expression in human cancer studies comparing cancer tissues with noncancerous tissues. Gene 527: 26-32, 2013.

38. Xu Q, Seeger FH, Castillo J, et al: Micro-RNA-34a contributes to the impaired function of bone marrow-derived mononuclear cells from patients with cardiovascular disease. J Am Coll Cardiol 59: 2107-2117, 2012

39. Ito T, Yagi S and Yamakuchi M: MicroRNA-34a regulation of endothelial senescence. Biochem Biophys Res Commun 398: 735-740, 2010.

40. Nishino J, Kim I, Chada K and Morrison SJ: Hmga2 promotes neural stem cell self-renewal in young but not old mice by reducing p16Ink4a and p19Arf Expression. Cell 135: 227-239, 2008.

41. Li S, Zhu J, Zhang W, et al: Signature microRNA expression profile of essential hypertension and its novel link to human cytomegalovirus infection. Circulation 124: 175-184, 2011.

42. Liu CG, Calin GA, Volinia S and Croce CM: MicroRNA expression profiling using microarrays. Nat Protoc 3: 563-578, 2008.

43. Brown BD, Gentner B, Cantore A, et al: Endogenous microRNA can be broadly exploited to regulate transgene expression according to tissue, lineage and differentiation state. Nat Biotechnol 25: 1457-1467, 2007.

44. Sarasin-Filipowicz M, Krol J, Markiewicz I, Heim MH and Filipowicz W: Decreased levels of microRNA miR-122 in individuals with hepatitis $C$ responding poorly to interferon therapy. Nat Med 15: 31-33, 2009.

45. Esuvaranathan K, Chiong E, Thamboo TP, et al: Predictive value of $\mathrm{p} 53$ and $\mathrm{pRb}$ expression in superficial bladder cancer patients treated with BCG and interferon-alpha. Cancer 109: 1097-1105, 2007.

46. Lu X, Wu L, Liu Z, Xie L and Wang S: Peripheral blood mononuclear cells inhibit proliferation and promote apoptosis of HeLa cells following stimulation with Bacillus Calmette-Guerin. Exp Ther Med 5: 561-566, 2013.

47. Kawaichi S: Cancer of bladder os spinal cord injury patients. In: Proceed. Ninth Ann Clin Cord Injury Conf pp 104-105, 1961

48. Silverman DT, Hartge P, Morrison AS and Devesa SS: Epidemiology of bladder cancer. Hematol Oncol Clin North Am 6: 1-30, 1992.

49. Burch JD, Rohan TE, Howe GR, et al: Risk of bladder cancer by source and type of tobacco exposure: A case-control study. Int J Cancer 44: 622-628, 1989. 